\title{
Method description
}

\section{Chemicals requirements}

- Control Plasma (CP), Level 1 \& Level 2, Recipe

- 1-tridecanoyl-2-hydroxy-sn-glycero-3-phosphocholine, LPC(13:0), Avanti Polar Lipids $(855476 \mathrm{P})$

- 1,2-dimyristoyl-sn-glycero-3-phosphocholine, PC(28:0), Avanti Polar Lipids (850345P)

- D3-Carnitine C2, Cambridge Isotope Laboratories (DLM-754-PK)

- D3-Carnitine C8, Cambridge Isotope Laboratories (DLM-755-0.01)

- D3-Carnitine C16, Cambridge Isotope Laboratories (DLM-1263-0.01)

- 13C6-D-Glucose, Cambridge Isotope Laboratories (CLM-1396)

- $\quad$ Riplate, 1.2 mL 96-deepwell, Carl Roth GmbH + Co. KG (EN06.1)

- 96 deepwell-plate, $1.0 \mathrm{~mL}$, polypropylene, Agilent (5042-6454)

- Ammonium acetate, Fluka (73594-25G-F)

- Water, HPLC grade, J.T. Backer

- Methanol, HPLC grade, CLN GmbH (CLN-8402.2500)

- Isopropanol, HPLC grade, LGC Standards (SO-3043-B040)

\section{$\underline{\text { Sample preparation }}$}

Proteins of $10 \mu \mathrm{L}$ plasma were precipitated by adding $500 \mu \mathrm{L}$ methanol, including 1-tridecanoyl2-hydroxy-sn-glycero-3-phosphocholine and 1,2-dimyristoyl-sn-glycero-3-phosphocholine as internal standards with a concentration of approximately $2 \mu \mathrm{mol} / \mathrm{L}$ and ammonium acetate with a concentration of $0.4 \mathrm{~g} / \mathrm{l}$. Samples were prepared using a $1.2 \mathrm{~mL}$ 96-deepwell plate. The plate was shaked for 30 minutes with $800 \mathrm{rpm}$ and afterwards cooled for 20 minutes at $-20^{\circ} \mathrm{C}$. After centrifugation at $4000 \mathrm{rpm}$ for 10 minutes $100 \mu \mathrm{L}$ of the supernatant were transferred in a $1.0 \mathrm{~mL} 96$ deepwell-plate, prefilled with $350 \mu \mathrm{L}$ methanol. 


\section{Mass spectrometric analyses}

After centrifugation, supernatants were used for flow-injection mass spectrometry analysis. The liquid chromatographic system (Agilent, Waldbronn, Germany) was coupled to a triple quadrupole mass spectrometer (QTRAP4000, Sciex, Darmstadt, Germany) with an electrospray ionization source. Analyses were split in two periods per sample each with $30 \mu \mathrm{L}$ injection volume to cover the full range of analytes. Samples were injected to $40 \mu \mathrm{L} / \mathrm{min}$ mobile phase with isocratic elution (76\% isopropanol, 19\% methanol and 5\% water) which results in broader peak elution than normal flow-injection analyses to ensure enough data points for the mass spectrometry analyses. Low flow rates were found to reduce lifetime of ESI needles, which would affect reproducibility and thus the flow was diluted by adding a further $200 \mu \mathrm{L} / \mathrm{min}$ mobile phase "post-column" via T-piece adapter to stabilize the electro spray for ionization. Mass spectrometric analysis was run in Multiple Reaction Monitoring mode. Period one was run with positive and negative ionisation and period two was run with positive ionisation.

\section{Quantification}

Quantification of metabolites has been done by comparison of signal-to-internal standard-ratios between samples and commercial available lyophilized aliquots of control plasma (Recipe, Germany). The concentrations of the control plasma were determined by AbsoluteIDQ p150 Kit from Biocrates ${ }^{\circledR}$, a previous published LC-MS/MS method (Uhl, JChormB, 2011) and by inhouse quantification with various standards. The entire analytical process was post-processed by Analyst 1.5.1 and the isotopomer correction for up to $\mathrm{M}+4$ was applied by $\mathrm{R}$ (programming language, version 3.0.1). 
Periode 1 - positive

\begin{tabular}{|c|c|c|c|c|c|c|}
\hline Q1 & Q3 & time & DP & EP & $\mathrm{CE}$ & CXP \\
\hline 162.1 & 85.1 & 20 & 61 & 10 & 27 & 15 \\
\hline 204.1 & 85.1 & 20 & 41 & 10 & 27 & 15 \\
\hline 206.1 & 85.1 & 20 & 41 & 10 & 27 & 15 \\
\hline 207.1 & 85.1 & 50 & 41 & 10 & 27 & 15 \\
\hline 216.1 & 85.1 & 20 & 49 & 10 & 27 & 15 \\
\hline 218.1 & 85.1 & 20 & 46 & 10 & 29 & 15 \\
\hline 230.1 & 85.1 & 20 & 52 & 10 & 29 & 15 \\
\hline 232.1 & 85.1 & 20 & 46 & 10 & 29 & 15 \\
\hline 234.1 & 85.1 & 20 & 53 & 10 & 30 & 15 \\
\hline 244.1 & 85.1 & 20 & 55 & 10 & 31 & 15 \\
\hline 246.1 & 85.1 & 20 & 46 & 10 & 29 & 15 \\
\hline 248.1 & 85.1 & 20 & 55 & 10 & 32 & 15 \\
\hline 258.1 & 85.1 & 20 & 57 & 10 & 33 & 15 \\
\hline 260.1 & 85.1 & 20 & 56 & 10 & 27 & 15 \\
\hline 262.1 & 85.1 & 20 & 58 & 10 & 33 & 15 \\
\hline 274.1 & 85.1 & 20 & 60 & 10 & 35 & 15 \\
\hline 276.2 & 85.1 & 20 & 61 & 10 & 35 & 15 \\
\hline 286.2 & 85.1 & 20 & 63 & 10 & 37 & 15 \\
\hline 288.2 & 85.1 & 20 & 66 & 10 & 33 & 15 \\
\hline 290.2 & 85.1 & 20 & 63 & 10 & 37 & 15 \\
\hline 291.2 & 85.1 & 50 & 66 & 10 & 33 & 15 \\
\hline 302.2 & 85.1 & 20 & 66 & 10 & 39 & 15 \\
\hline 304.2 & 85.1 & 20 & 66 & 10 & 39 & 15 \\
\hline 312.2 & 85.1 & 20 & 67 & 10 & 40 & 15 \\
\hline 314.2 & 85.1 & 20 & 68 & 10 & 40 & 15 \\
\hline 316.2 & 85.1 & 20 & 56 & 10 & 37 & 15 \\
\hline 342.3 & 85.1 & 20 & 73 & 10 & 44 & 15 \\
\hline 344.3 & 85.1 & 20 & 73 & 10 & 44 & 15 \\
\hline 368.3 & 85.1 & 20 & 78 & 10 & 47 & 15 \\
\hline 370.3 & 85.1 & 20 & 78 & 10 & 47 & 15 \\
\hline 372.3 & 85.1 & 20 & 86 & 10 & 45 & 15 \\
\hline 374.3 & 85.1 & 20 & 86 & 10 & 45 & 15 \\
\hline 384.3 & 85.1 & 20 & 81 & 10 & 49 & 15 \\
\hline 386.3 & 85.1 & 20 & 81 & 10 & 50 & 15 \\
\hline 396.3 & 85.1 & 20 & 83 & 10 & 51 & 15 \\
\hline 398.3 & 85.1 & 20 & 84 & 10 & 51 & 15 \\
\hline 400.3 & 85.1 & 20 & 84 & 10 & 51 & 15 \\
\hline 402.3 & 85.1 & 20 & 84 & 10 & 51 & 15 \\
\hline 403.3 & 85.1 & 50 & 84 & 10 & 51 & 15 \\
\hline 412.3 & 85.1 & 20 & 86 & 10 & 53 & 15 \\
\hline
\end{tabular}




\begin{tabular}{|c|c|c|c|c|c|c|}
\hline 414.3 & 85.1 & 20 & 87 & 10 & 53 & 15 \\
\hline 416.3 & 85.1 & 20 & 87 & 10 & 53 & 15 \\
\hline 420.3 & 85.1 & 20 & 89 & 10 & 54 & 15 \\
\hline 422.3 & 85.1 & 20 & 89 & 10 & 54 & 15 \\
\hline 424.3 & 85.1 & 20 & 89 & 10 & 54 & 15 \\
\hline 426.3 & 85.1 & 20 & 89 & 10 & 55 & 15 \\
\hline 428.3 & 85.1 & 20 & 94 & 10 & 63 & 15 \\
\hline 440.3 & 85.1 & 20 & 94 & 10 & 65 & 15 \\
\hline 442.3 & 85.1 & 20 & 94 & 10 & 65 & 15 \\
\hline 446.3 & 85.1 & 20 & 96 & 10 & 65 & 15 \\
\hline 448.3 & 85.1 & 20 & 96 & 10 & 65 & 15 \\
\hline 450.3 & 85.1 & 20 & 96 & 10 & 65 & 15 \\
\hline 452.3 & 85.1 & 20 & 96 & 10 & 65 & 15 \\
\hline 454.3 & 85.1 & 20 & 96 & 10 & 65 & 15 \\
\hline 456.3 & 85.1 & 20 & 96 & 10 & 65 & 15 \\
\hline 472.3 & 85.1 & 20 & 96 & 10 & 65 & 15 \\
\hline 474.3 & 85.1 & 20 & 96 & 10 & 65 & 15 \\
\hline 476.3 & 85.1 & 20 & 96 & 10 & 65 & 15 \\
\hline 478.3 & 85.1 & 20 & 96 & 10 & 65 & 15 \\
\hline 480.3 & 85.1 & 20 & 96 & 10 & 65 & 15 \\
\hline 482.3 & 85.1 & 20 & 96 & 10 & 65 & 15 \\
\hline 484.3 & 85.1 & 20 & 96 & 10 & 65 & 15 \\
\hline 449.3 & 184 & 20 & 79 & 10 & 30 & 10 \\
\hline 450.3 & 184 & 20 & 79 & 10 & 30 & 10 \\
\hline 451.3 & 184 & 20 & 79 & 10 & 30 & 10 \\
\hline 452.3 & 184 & 20 & 79 & 10 & 30 & 10 \\
\hline 453.3 & 184 & 20 & 79 & 10 & 30 & 10 \\
\hline 454.3 & 184 & 50 & 79 & 10 & 30 & 10 \\
\hline 455.3 & 184 & 20 & 79 & 10 & 30 & 10 \\
\hline 456.3 & 184 & 20 & 79 & 10 & 30 & 10 \\
\hline 457.3 & 184 & 20 & 79 & 10 & 30 & 10 \\
\hline 458.3 & 184 & 20 & 79 & 10 & 30 & 10 \\
\hline 459.3 & 184 & 20 & 79 & 10 & 30 & 10 \\
\hline 460.3 & 184 & 20 & 79 & 10 & 30 & 10 \\
\hline 461.3 & 184 & 20 & 79 & 10 & 30 & 10 \\
\hline 462.3 & 184 & 20 & 79 & 10 & 30 & 10 \\
\hline 463.3 & 184 & 20 & 79 & 10 & 30 & 10 \\
\hline 464.3 & 184 & 20 & 79 & 10 & 30 & 10 \\
\hline 465.3 & 184 & 20 & 79 & 10 & 30 & 10 \\
\hline 466.3 & 184 & 20 & 79 & 10 & 30 & 10 \\
\hline 467.3 & 184 & 20 & 79 & 10 & 30 & 10 \\
\hline 468.3 & 184 & 20 & 79 & 10 & 30 & 10 \\
\hline 469.3 & 184 & 20 & 79 & 10 & 30 & 10 \\
\hline 470.3 & 184 & 20 & 79 & 10 & 30 & 10 \\
\hline
\end{tabular}




\begin{tabular}{|c|c|c|c|c|c|c|}
\hline 471.3 & 184 & 20 & 79 & 10 & 30 & 10 \\
\hline 472.3 & 184 & 20 & 79 & 10 & 30 & 10 \\
\hline 473.3 & 184 & 20 & 79 & 10 & 30 & 10 \\
\hline 474.3 & 184 & 20 & 79 & 10 & 30 & 10 \\
\hline 475.3 & 184 & 20 & 79 & 10 & 30 & 10 \\
\hline 476.3 & 184 & 20 & 79 & 10 & 30 & 10 \\
\hline 477.3 & 184 & 20 & 79 & 10 & 30 & 10 \\
\hline 478.3 & 184 & 20 & 79 & 10 & 30 & 10 \\
\hline 479.3 & 184 & 20 & 79 & 10 & 30 & 10 \\
\hline 480.3 & 184 & 20 & 79 & 10 & 30 & 10 \\
\hline 481.3 & 184 & 20 & 79 & 10 & 30 & 10 \\
\hline 482.3 & 184 & 20 & 79 & 10 & 30 & 10 \\
\hline 483.3 & 184 & 20 & 79 & 10 & 30 & 10 \\
\hline 484.3 & 184 & 20 & 79 & 10 & 30 & 10 \\
\hline 485.3 & 184 & 20 & 79 & 10 & 30 & 10 \\
\hline 486.3 & 184 & 20 & 79 & 10 & 30 & 10 \\
\hline 487.3 & 184 & 20 & 79 & 10 & 30 & 10 \\
\hline 488.3 & 184 & 20 & 79 & 10 & 30 & 10 \\
\hline 489.3 & 184 & 20 & 79 & 10 & 30 & 10 \\
\hline 490.3 & 184 & 20 & 79 & 10 & 30 & 10 \\
\hline 491.3 & 184 & 20 & 79 & 10 & 30 & 10 \\
\hline 492.3 & 184 & 20 & 79 & 10 & 30 & 10 \\
\hline 493.3 & 184 & 20 & 79 & 10 & 30 & 10 \\
\hline 494.3 & 184 & 20 & 79 & 10 & 30 & 10 \\
\hline 495.3 & 184 & 20 & 79 & 10 & 30 & 10 \\
\hline 496.3 & 184 & 20 & 81 & 10 & 31 & 10 \\
\hline 497.3 & 184 & 20 & 81 & 10 & 31 & 10 \\
\hline 498.3 & 184 & 20 & 81 & 10 & 31 & 10 \\
\hline 499.3 & 184 & 20 & 81 & 10 & 31 & 10 \\
\hline 500.3 & 184 & 20 & 81 & 10 & 31 & 10 \\
\hline 501.3 & 184 & 20 & 81 & 10 & 31 & 10 \\
\hline 502.3 & 184 & 20 & 81 & 10 & 31 & 10 \\
\hline 503.3 & 184 & 20 & 81 & 10 & 31 & 10 \\
\hline 504.3 & 184 & 20 & 81 & 10 & 31 & 10 \\
\hline 505.3 & 184 & 20 & 81 & 10 & 31 & 10 \\
\hline 506.3 & 184 & 20 & 81 & 10 & 31 & 10 \\
\hline 507.3 & 184 & 20 & 81 & 10 & 31 & 10 \\
\hline 508.3 & 184 & 20 & 81 & 10 & 31 & 10 \\
\hline 509.3 & 184 & 20 & 81 & 10 & 31 & 10 \\
\hline 510.3 & 184 & 20 & 82 & 10 & 31 & 10 \\
\hline 511.3 & 184 & 20 & 82 & 10 & 31 & 10 \\
\hline 512.3 & 184 & 20 & 82 & 10 & 31 & 10 \\
\hline 513.3 & 184 & 20 & 82 & 10 & 31 & 10 \\
\hline 514.3 & 184 & 20 & 82 & 10 & 31 & 10 \\
\hline
\end{tabular}




\begin{tabular}{|c|c|c|c|c|c|c|}
\hline 515.3 & 184 & 20 & 82 & 10 & 31 & 10 \\
\hline 516.3 & 184 & 20 & 82 & 10 & 31 & 10 \\
\hline 517.3 & 184 & 20 & 82 & 10 & 31 & 10 \\
\hline 518.3 & 184 & 20 & 82 & 10 & 31 & 10 \\
\hline 519.3 & 184 & 20 & 82 & 10 & 31 & 10 \\
\hline 520.3 & 184 & 20 & 82 & 10 & 31 & 10 \\
\hline 521.3 & 184 & 20 & 82 & 10 & 31 & 10 \\
\hline 522.3 & 184 & 20 & 82 & 10 & 31 & 10 \\
\hline 523.3 & 184 & 20 & 82 & 10 & 31 & 10 \\
\hline 524.3 & 184 & 20 & 85 & 10 & 31 & 10 \\
\hline 525.3 & 184 & 20 & 85 & 10 & 31 & 10 \\
\hline 526.3 & 184 & 20 & 85 & 10 & 31 & 10 \\
\hline 527.3 & 184 & 20 & 85 & 10 & 31 & 10 \\
\hline 528.3 & 184 & 20 & 85 & 10 & 31 & 10 \\
\hline 529.3 & 184 & 20 & 85 & 10 & 31 & 10 \\
\hline 530.3 & 184 & 20 & 85 & 10 & 31 & 10 \\
\hline 531.3 & 184 & 20 & 85 & 10 & 31 & 10 \\
\hline 532.3 & 184 & 20 & 85 & 10 & 31 & 10 \\
\hline 533.3 & 184 & 20 & 85 & 10 & 31 & 10 \\
\hline 534.3 & 184 & 20 & 85 & 10 & 31 & 10 \\
\hline 535.3 & 184 & 20 & 85 & 10 & 31 & 10 \\
\hline 536.3 & 184 & 20 & 85 & 10 & 31 & 10 \\
\hline 537.3 & 184 & 20 & 85 & 10 & 31 & 10 \\
\hline 538.3 & 184 & 20 & 85 & 10 & 31 & 10 \\
\hline 539.3 & 184 & 20 & 85 & 10 & 31 & 10 \\
\hline 540.3 & 184 & 20 & 85 & 10 & 31 & 10 \\
\hline 541.3 & 184 & 20 & 85 & 10 & 31 & 10 \\
\hline 542.3 & 184 & 20 & 85 & 10 & 31 & 10 \\
\hline 543.3 & 184 & 20 & 85 & 10 & 31 & 10 \\
\hline 544.3 & 184 & 20 & 85 & 10 & 31 & 10 \\
\hline 545.3 & 184 & 20 & 85 & 10 & 31 & 10 \\
\hline 546.3 & 184 & 20 & 87 & 10 & 31 & 10 \\
\hline 547.3 & 184 & 20 & 87 & 10 & 31 & 10 \\
\hline 548.3 & 184 & 20 & 87 & 10 & 31 & 10 \\
\hline 549.3 & 184 & 20 & 87 & 10 & 31 & 10 \\
\hline 550.3 & 184 & 20 & 87 & 10 & 31 & 10 \\
\hline 551.3 & 184 & 20 & 87 & 10 & 31 & 10 \\
\hline 552.3 & 184 & 20 & 87 & 10 & 31 & 10 \\
\hline 553.3 & 184 & 20 & 87 & 10 & 31 & 10 \\
\hline 554.3 & 184 & 20 & 87 & 10 & 31 & 10 \\
\hline 555.3 & 184 & 20 & 87 & 10 & 31 & 10 \\
\hline 556.3 & 184 & 20 & 87 & 10 & 31 & 10 \\
\hline 557.3 & 184 & 20 & 87 & 10 & 31 & 10 \\
\hline 558.3 & 184 & 20 & 87 & 10 & 31 & 10 \\
\hline
\end{tabular}




$\begin{array}{lllllll}559.3 & 184 & 20 & 87 & 10 & 31 & 10 \\ 560.3 & 184 & 20 & 87 & 10 & 31 & 10 \\ 561.3 & 184 & 20 & 87 & 10 & 31 & 10 \\ 562.3 & 184 & 20 & 87 & 10 & 31 & 10 \\ 563.3 & 184 & 20 & 87 & 10 & 31 & 10 \\ 564.3 & 184 & 20 & 87 & 10 & 31 & 10 \\ 565.3 & 184 & 20 & 87 & 10 & 31 & 10 \\ 566.3 & 184 & 20 & 87 & 10 & 31 & 10 \\ 567.4 & 184 & 20 & 94 & 10 & 32 & 10 \\ 568.4 & 184 & 20 & 94 & 10 & 32 & 10 \\ 569.4 & 184 & 20 & 94 & 10 & 32 & 10 \\ 570.4 & 184 & 20 & 94 & 10 & 32 & 10 \\ 571.4 & 184 & 20 & 94 & 10 & 32 & 10 \\ 572.4 & 184 & 20 & 94 & 10 & 32 & 10 \\ 573.4 & 184 & 20 & 94 & 10 & 32 & 10 \\ 574.4 & 184 & 20 & 94 & 10 & 32 & 10 \\ 575.4 & 184 & 20 & 94 & 10 & 32 & 10 \\ 576.4 & 184 & 20 & 94 & 10 & 32 & 10 \\ 577.4 & 184 & 20 & 94 & 10 & 32 & 10 \\ 578.4 & 184 & 20 & 94 & 10 & 32 & 10 \\ 579.4 & 184 & 20 & 94 & 10 & 32 & 10 \\ 580.4 & 184 & 20 & 94 & 10 & 32 & 10\end{array}$

Period 1 - negative

$\begin{array}{ccccccc}\text { Q1 } & \text { Q3 } & \text { time } & \text { DP } & \text { EP } & \text { CE } & \text { CXP } \\ 179.05 & 89 & 50 & -55 & -10 & -12 & -15 \\ 185.05 & 92 & 50 & -55 & -10 & -12 & -15\end{array}$

Period 2 - positive

$\begin{array}{ccccccc}\text { Q1 } & \text { Q3 } & \text { time } & \text { DP } & \text { EP } & \text { CE } & \text { CXP } \\ 581.4 & 184 & 20 & 94 & 10 & 32 & 10 \\ 582.4 & 184 & 20 & 94 & 10 & 32 & 10 \\ 583.4 & 184 & 20 & 94 & 10 & 32 & 10 \\ 584.4 & 184 & 20 & 94 & 10 & 32 & 10 \\ 585.4 & 184 & 20 & 94 & 10 & 32 & 10 \\ 586.4 & 184 & 20 & 94 & 10 & 32 & 10 \\ 587.4 & 184 & 20 & 94 & 10 & 32 & 10 \\ 588.4 & 184 & 20 & 94 & 10 & 32 & 10 \\ 589.4 & 184 & 20 & 94 & 10 & 32 & 10\end{array}$




\begin{tabular}{|c|c|c|c|c|c|c|}
\hline 590.4 & 184 & 20 & 94 & 10 & 32 & 10 \\
\hline 591.4 & 184 & 20 & 94 & 10 & 32 & 10 \\
\hline 592.4 & 184 & 20 & 94 & 10 & 32 & 10 \\
\hline 593.4 & 184 & 20 & 94 & 10 & 32 & 10 \\
\hline 594.4 & 184 & 20 & 94 & 10 & 32 & 10 \\
\hline 595.4 & 184 & 20 & 94 & 10 & 32 & 10 \\
\hline 596.4 & 184 & 20 & 94 & 10 & 32 & 10 \\
\hline 597.4 & 184 & 20 & 94 & 10 & 32 & 10 \\
\hline 598.4 & 184 & 20 & 94 & 10 & 32 & 10 \\
\hline 599.4 & 184 & 20 & 94 & 10 & 32 & 10 \\
\hline 600.4 & 184 & 20 & 94 & 10 & 32 & 10 \\
\hline 601.4 & 184 & 20 & 94 & 10 & 32 & 10 \\
\hline 602.4 & 184 & 20 & 94 & 10 & 32 & 10 \\
\hline 603.4 & 184 & 20 & 94 & 10 & 32 & 10 \\
\hline 604.4 & 184 & 20 & 94 & 10 & 32 & 10 \\
\hline 605.4 & 184 & 20 & 94 & 10 & 32 & 10 \\
\hline 606.4 & 184 & 20 & 94 & 10 & 32 & 10 \\
\hline 607.4 & 184 & 20 & 94 & 10 & 32 & 10 \\
\hline 608.4 & 184 & 20 & 94 & 10 & 32 & 10 \\
\hline 609.4 & 184 & 20 & 94 & 10 & 32 & 10 \\
\hline 610.4 & 184 & 20 & 94 & 10 & 32 & 10 \\
\hline 611.4 & 184 & 20 & 94 & 10 & 32 & 10 \\
\hline 612.4 & 184 & 20 & 94 & 10 & 32 & 10 \\
\hline 613.4 & 184 & 20 & 94 & 10 & 32 & 10 \\
\hline 614.4 & 184 & 20 & 94 & 10 & 32 & 10 \\
\hline 615.4 & 184 & 20 & 94 & 10 & 32 & 10 \\
\hline 616.4 & 184 & 20 & 94 & 10 & 32 & 10 \\
\hline 617.4 & 184 & 20 & 94 & 10 & 32 & 10 \\
\hline 618.4 & 184 & 20 & 94 & 10 & 32 & 10 \\
\hline 619.4 & 184 & 20 & 94 & 10 & 32 & 10 \\
\hline 620.4 & 184 & 20 & 94 & 10 & 32 & 10 \\
\hline 621.4 & 184 & 20 & 96 & 10 & 33 & 10 \\
\hline 622.4 & 184 & 20 & 98 & 10 & 33 & 10 \\
\hline 623.4 & 184 & 20 & 98 & 10 & 33 & 10 \\
\hline 624.4 & 184 & 20 & 98 & 10 & 33 & 10 \\
\hline 625.4 & 184 & 20 & 98 & 10 & 33 & 10 \\
\hline 626.4 & 184 & 20 & 98 & 10 & 33 & 10 \\
\hline 627.4 & 184 & 20 & 98 & 10 & 33 & 10 \\
\hline 628.4 & 184 & 20 & 98 & 10 & 33 & 10 \\
\hline 629.4 & 184 & 20 & 98 & 10 & 33 & 10 \\
\hline 630.4 & 184 & 20 & 98 & 10 & 33 & 10 \\
\hline 631.4 & 184 & 20 & 98 & 10 & 33 & 10 \\
\hline 632.4 & 184 & 20 & 98 & 10 & 33 & 10 \\
\hline 633.4 & 184 & 20 & 98 & 10 & 33 & 10 \\
\hline
\end{tabular}




\begin{tabular}{|c|c|c|c|c|c|c|}
\hline 634.4 & 184 & 20 & 98 & 10 & 33 & 10 \\
\hline 635.4 & 184 & 20 & 98 & 10 & 33 & 10 \\
\hline 636.5 & 184 & 20 & 101 & 10 & 34 & 10 \\
\hline 637.5 & 184 & 20 & 101 & 10 & 34 & 10 \\
\hline 638.5 & 184 & 20 & 101 & 10 & 34 & 10 \\
\hline 639.5 & 184 & 20 & 101 & 10 & 34 & 10 \\
\hline 640.5 & 184 & 20 & 101 & 10 & 34 & 10 \\
\hline 641.5 & 184 & 20 & 101 & 10 & 34 & 10 \\
\hline 642.5 & 184 & 20 & 101 & 10 & 34 & 10 \\
\hline 643.5 & 184 & 20 & 101 & 10 & 34 & 10 \\
\hline 644.5 & 184 & 20 & 101 & 10 & 34 & 10 \\
\hline 645.5 & 184 & 20 & 101 & 10 & 34 & 10 \\
\hline 646.5 & 184 & 20 & 101 & 10 & 34 & 10 \\
\hline 647.5 & 184 & 20 & 101 & 10 & 34 & 10 \\
\hline 648.5 & 184 & 20 & 101 & 10 & 34 & 10 \\
\hline 649.5 & 184 & 20 & 101 & 10 & 34 & 10 \\
\hline 650.5 & 184 & 20 & 103 & 10 & 35 & 10 \\
\hline 651.5 & 184 & 20 & 103 & 10 & 35 & 10 \\
\hline 652.5 & 184 & 20 & 103 & 10 & 35 & 10 \\
\hline 653.5 & 184 & 20 & 103 & 10 & 35 & 10 \\
\hline 654.5 & 184 & 20 & 103 & 10 & 35 & 10 \\
\hline 655.5 & 184 & 20 & 103 & 10 & 35 & 10 \\
\hline 656.5 & 184 & 20 & 103 & 10 & 35 & 10 \\
\hline 657.5 & 184 & 20 & 103 & 10 & 35 & 10 \\
\hline 658.5 & 184 & 20 & 103 & 10 & 35 & 10 \\
\hline 659.5 & 184 & 20 & 103 & 10 & 35 & 10 \\
\hline 660.5 & 184 & 20 & 103 & 10 & 35 & 10 \\
\hline 661.5 & 184 & 20 & 103 & 10 & 35 & 10 \\
\hline 662.5 & 184 & 20 & 103 & 10 & 35 & 10 \\
\hline 663.5 & 184 & 20 & 103 & 10 & 35 & 10 \\
\hline 664.5 & 184 & 20 & 105 & 10 & 35 & 10 \\
\hline 665.5 & 184 & 20 & 105 & 10 & 35 & 10 \\
\hline 666.5 & 184 & 20 & 105 & 10 & 35 & 10 \\
\hline 667.5 & 184 & 20 & 105 & 10 & 35 & 10 \\
\hline 668.5 & 184 & 20 & 105 & 10 & 35 & 10 \\
\hline 669.5 & 184 & 20 & 105 & 10 & 35 & 10 \\
\hline 670.5 & 184 & 20 & 105 & 10 & 35 & 10 \\
\hline 671.5 & 184 & 20 & 105 & 10 & 35 & 10 \\
\hline 672.5 & 184 & 20 & 105 & 10 & 35 & 10 \\
\hline 673.5 & 184 & 20 & 105 & 10 & 35 & 10 \\
\hline 674.5 & 184 & 20 & 105 & 10 & 35 & 10 \\
\hline 675.5 & 184 & 20 & 105 & 10 & 35 & 10 \\
\hline 676.5 & 184 & 20 & 106 & 10 & 35 & 10 \\
\hline 677.5 & 184 & 20 & 106 & 10 & 35 & 10 \\
\hline
\end{tabular}




\begin{tabular}{|c|c|c|c|c|c|c|}
\hline 678.5 & 184 & 50 & 108 & 10 & 36 & 10 \\
\hline 679.5 & 184 & 20 & 108 & 10 & 36 & 10 \\
\hline 680.5 & 184 & 20 & 108 & 10 & 36 & 10 \\
\hline 681.5 & 184 & 20 & 108 & 10 & 36 & 10 \\
\hline 682.5 & 184 & 20 & 108 & 10 & 36 & 10 \\
\hline 683.5 & 184 & 20 & 108 & 10 & 36 & 10 \\
\hline 684.5 & 184 & 20 & 108 & 10 & 36 & 10 \\
\hline 685.5 & 184 & 20 & 108 & 10 & 36 & 10 \\
\hline 686.5 & 184 & 20 & 108 & 10 & 36 & 10 \\
\hline 687.5 & 184 & 20 & 108 & 10 & 36 & 10 \\
\hline 688.5 & 184 & 20 & 108 & 10 & 36 & 10 \\
\hline 689.5 & 184 & 20 & 108 & 10 & 36 & 10 \\
\hline 690.5 & 184 & 20 & 109 & 10 & 36 & 10 \\
\hline 691.5 & 184 & 20 & 109 & 10 & 36 & 10 \\
\hline 692.5 & 184 & 20 & 109 & 10 & 36 & 10 \\
\hline 693.5 & 184 & 20 & 109 & 10 & 36 & 10 \\
\hline 694.5 & 184 & 20 & 109 & 10 & 36 & 10 \\
\hline 695.5 & 184 & 20 & 109 & 10 & 36 & 10 \\
\hline 696.5 & 184 & 20 & 109 & 10 & 36 & 10 \\
\hline 697.5 & 184 & 20 & 109 & 10 & 36 & 10 \\
\hline 698.5 & 184 & 20 & 109 & 10 & 36 & 10 \\
\hline 699.5 & 184 & 20 & 109 & 10 & 36 & 10 \\
\hline 700.5 & 184 & 20 & 109 & 10 & 36 & 10 \\
\hline 701.5 & 184 & 20 & 111 & 10 & 37 & 10 \\
\hline 702.5 & 184 & 20 & 111 & 10 & 37 & 10 \\
\hline 703.5 & 184 & 20 & 112 & 10 & 37 & 10 \\
\hline 704.5 & 184 & 20 & 112 & 10 & 37 & 10 \\
\hline 705.5 & 184 & 20 & 112 & 10 & 37 & 10 \\
\hline 706.5 & 184 & 20 & 114 & 10 & 37 & 10 \\
\hline 707.5 & 184 & 20 & 114 & 10 & 37 & 10 \\
\hline 708.5 & 184 & 20 & 114 & 10 & 37 & 10 \\
\hline 709.5 & 184 & 20 & 114 & 10 & 37 & 10 \\
\hline 710.5 & 184 & 20 & 114 & 10 & 37 & 10 \\
\hline 711.5 & 184 & 20 & 114 & 10 & 37 & 10 \\
\hline 712.5 & 184 & 20 & 114 & 10 & 37 & 10 \\
\hline 713.5 & 184 & 20 & 114 & 10 & 37 & 10 \\
\hline 714.5 & 184 & 20 & 114 & 10 & 37 & 10 \\
\hline 715.5 & 184 & 20 & 114 & 10 & 37 & 10 \\
\hline 716.5 & 184 & 20 & 114 & 10 & 37 & 10 \\
\hline 717.5 & 184 & 20 & 114 & 10 & 38 & 10 \\
\hline 718.5 & 184 & 20 & 115 & 10 & 38 & 10 \\
\hline 719.5 & 184 & 20 & 115 & 10 & 38 & 10 \\
\hline 720.5 & 184 & 20 & 115 & 10 & 38 & 10 \\
\hline 721.5 & 184 & 20 & 115 & 10 & 38 & 10 \\
\hline
\end{tabular}




\begin{tabular}{|c|c|c|c|c|c|c|}
\hline 722.5 & 184 & 20 & 115 & 10 & 38 & 10 \\
\hline 723.5 & 184 & 20 & 115 & 10 & 38 & 10 \\
\hline 724.5 & 184 & 20 & 115 & 10 & 38 & 10 \\
\hline 725.6 & 184 & 20 & 115 & 10 & 38 & 10 \\
\hline 726.6 & 184 & 20 & 115 & 10 & 38 & 10 \\
\hline 727.6 & 184 & 20 & 115 & 10 & 38 & 10 \\
\hline 728.6 & 184 & 20 & 117 & 10 & 38 & 10 \\
\hline 729.6 & 184 & 20 & 117 & 10 & 38 & 10 \\
\hline 730.6 & 184 & 20 & 117 & 10 & 38 & 10 \\
\hline 731.6 & 184 & 20 & 117 & 10 & 38 & 10 \\
\hline 732.6 & 184 & 20 & 118 & 10 & 39 & 10 \\
\hline 733.6 & 184 & 20 & 118 & 10 & 39 & 10 \\
\hline 734.6 & 184 & 20 & 119 & 10 & 39 & 10 \\
\hline 735.6 & 184 & 20 & 119 & 10 & 39 & 10 \\
\hline 736.6 & 184 & 20 & 119 & 10 & 39 & 10 \\
\hline 737.6 & 184 & 20 & 119 & 10 & 39 & 10 \\
\hline 738.6 & 184 & 20 & 119 & 10 & 39 & 10 \\
\hline 739.6 & 184 & 20 & 119 & 10 & 39 & 10 \\
\hline 740.6 & 184 & 20 & 119 & 10 & 39 & 10 \\
\hline 741.6 & 184 & 20 & 119 & 10 & 39 & 10 \\
\hline 742.6 & 184 & 20 & 120 & 10 & 39 & 10 \\
\hline 743.6 & 184 & 20 & 120 & 10 & 39 & 10 \\
\hline 744.6 & 184 & 20 & 120 & 10 & 39 & 10 \\
\hline 745.6 & 184 & 20 & 120 & 10 & 39 & 10 \\
\hline 746.6 & 184 & 20 & 121 & 10 & 39 & 10 \\
\hline 747.6 & 184 & 20 & 121 & 10 & 39 & 10 \\
\hline 748.6 & 184 & 20 & 122 & 10 & 40 & 10 \\
\hline 749.6 & 184 & 20 & 122 & 10 & 40 & 10 \\
\hline 750.6 & 184 & 20 & 122 & 10 & 40 & 10 \\
\hline 751.6 & 184 & 20 & 122 & 10 & 40 & 10 \\
\hline 752.6 & 184 & 20 & 122 & 10 & 40 & 10 \\
\hline 753.6 & 184 & 20 & 122 & 10 & 40 & 10 \\
\hline 754.6 & 184 & 20 & 122 & 10 & 40 & 10 \\
\hline 755.6 & 184 & 20 & 123 & 10 & 40 & 10 \\
\hline 756.6 & 184 & 20 & 123 & 10 & 40 & 10 \\
\hline 757.6 & 184 & 20 & 123 & 10 & 40 & 10 \\
\hline 758.6 & 184 & 20 & 123 & 10 & 40 & 10 \\
\hline 759.6 & 184 & 20 & 123 & 10 & 40 & 10 \\
\hline 760.6 & 184 & 20 & 125 & 10 & 41 & 10 \\
\hline 761.6 & 184 & 20 & 125 & 10 & 41 & 10 \\
\hline 762.6 & 184 & 20 & 125 & 10 & 41 & 10 \\
\hline 763.6 & 184 & 20 & 125 & 10 & 41 & 10 \\
\hline 764.6 & 184 & 20 & 125 & 10 & 41 & 10 \\
\hline 765.6 & 184 & 20 & 125 & 10 & 41 & 10 \\
\hline
\end{tabular}




\begin{tabular}{|c|c|c|c|c|c|c|}
\hline 766.6 & 184 & 20 & 125 & 10 & 41 & 10 \\
\hline 767.6 & 184 & 20 & 125 & 10 & 41 & 10 \\
\hline 768.6 & 184 & 20 & 126 & 10 & 41 & 10 \\
\hline 769.6 & 184 & 20 & 126 & 10 & 41 & 10 \\
\hline 770.6 & 184 & 20 & 126 & 10 & 41 & 10 \\
\hline 771.6 & 184 & 20 & 126 & 10 & 41 & 10 \\
\hline 772.6 & 184 & 20 & 127 & 10 & 41 & 10 \\
\hline 773.6 & 184 & 20 & 127 & 10 & 41 & 10 \\
\hline 774.6 & 184 & 20 & 127 & 10 & 41 & 10 \\
\hline 775.6 & 184 & 20 & 127 & 10 & 41 & 10 \\
\hline 776.6 & 184 & 20 & 128 & 10 & 42 & 10 \\
\hline 777.6 & 184 & 20 & 128 & 10 & 42 & 10 \\
\hline 778.6 & 184 & 20 & 128 & 10 & 42 & 10 \\
\hline 779.6 & 184 & 20 & 128 & 10 & 42 & 10 \\
\hline 780.6 & 184 & 20 & 128 & 10 & 42 & 10 \\
\hline 781.6 & 184 & 20 & 129 & 10 & 42 & 10 \\
\hline 782.6 & 184 & 20 & 129 & 10 & 42 & 10 \\
\hline 783.6 & 184 & 20 & 129 & 10 & 42 & 10 \\
\hline 784.6 & 184 & 20 & 130 & 10 & 42 & 10 \\
\hline 785.6 & 184 & 20 & 130 & 10 & 42 & 10 \\
\hline 786.6 & 184 & 20 & 130 & 10 & 42 & 10 \\
\hline 787.6 & 184 & 20 & 130 & 10 & 42 & 10 \\
\hline 788.6 & 184 & 20 & 131 & 10 & 43 & 10 \\
\hline 789.6 & 184 & 20 & 131 & 10 & 43 & 10 \\
\hline 790.6 & 184 & 20 & 131 & 10 & 43 & 10 \\
\hline 791.6 & 184 & 20 & 131 & 10 & 43 & 10 \\
\hline 792.6 & 184 & 20 & 132 & 10 & 43 & 10 \\
\hline 793.6 & 184 & 20 & 132 & 10 & 43 & 10 \\
\hline 794.6 & 184 & 20 & 132 & 10 & 43 & 10 \\
\hline 795.6 & 184 & 20 & 132 & 10 & 43 & 10 \\
\hline 796.6 & 184 & 20 & 133 & 10 & 43 & 10 \\
\hline 797.6 & 184 & 20 & 133 & 10 & 43 & 10 \\
\hline 798.6 & 184 & 20 & 133 & 10 & 43 & 10 \\
\hline 799.6 & 184 & 20 & 133 & 10 & 43 & 10 \\
\hline 800.6 & 184 & 20 & 133 & 10 & 43 & 10 \\
\hline 801.6 & 184 & 20 & 134 & 10 & 43 & 10 \\
\hline 802.6 & 184 & 20 & 134 & 10 & 44 & 10 \\
\hline 803.6 & 184 & 20 & 134 & 10 & 44 & 10 \\
\hline 804.6 & 184 & 20 & 135 & 10 & 44 & 10 \\
\hline 805.6 & 184 & 20 & 135 & 10 & 44 & 10 \\
\hline 806.6 & 184 & 20 & 135 & 10 & 44 & 10 \\
\hline 807.6 & 184 & 20 & 135 & 10 & 44 & 10 \\
\hline 808.6 & 184 & 20 & 136 & 10 & 44 & 10 \\
\hline 809.7 & 184 & 20 & 136 & 10 & 44 & 10 \\
\hline
\end{tabular}




\begin{tabular}{|c|c|c|c|c|c|c|}
\hline 810.7 & 184 & 20 & 136 & 10 & 44 & 10 \\
\hline 811.7 & 184 & 20 & 136 & 10 & 44 & 10 \\
\hline 812.7 & 184 & 20 & 136 & 10 & 44 & 10 \\
\hline 813.7 & 184 & 20 & 137 & 10 & 45 & 10 \\
\hline 814.7 & 184 & 20 & 137 & 10 & 45 & 10 \\
\hline 815.7 & 184 & 20 & 137 & 10 & 45 & 10 \\
\hline 816.7 & 184 & 20 & 138 & 10 & 45 & 10 \\
\hline 817.7 & 184 & 20 & 138 & 10 & 45 & 10 \\
\hline 818.7 & 184 & 20 & 138 & 10 & 45 & 10 \\
\hline 819.7 & 184 & 20 & 138 & 10 & 45 & 10 \\
\hline 820.7 & 184 & 20 & 139 & 10 & 45 & 10 \\
\hline 821.7 & 184 & 20 & 139 & 10 & 45 & 10 \\
\hline 822.7 & 184 & 20 & 139 & 10 & 45 & 10 \\
\hline 823.7 & 184 & 20 & 139 & 10 & 45 & 10 \\
\hline 824.7 & 184 & 20 & 140 & 10 & 45 & 10 \\
\hline 825.7 & 184 & 20 & 140 & 10 & 45 & 10 \\
\hline 826.7 & 184 & 20 & 140 & 10 & 46 & 10 \\
\hline 827.7 & 184 & 20 & 140 & 10 & 46 & 10 \\
\hline 828.7 & 184 & 20 & 141 & 10 & 46 & 10 \\
\hline 829.7 & 184 & 20 & 141 & 10 & 46 & 10 \\
\hline 830.7 & 184 & 20 & 141 & 10 & 46 & 10 \\
\hline 831.7 & 184 & 20 & 141 & 10 & 46 & 10 \\
\hline 832.7 & 184 & 20 & 142 & 10 & 46 & 10 \\
\hline 833.7 & 184 & 20 & 142 & 10 & 46 & 10 \\
\hline 834.7 & 184 & 20 & 143 & 10 & 46 & 10 \\
\hline 835.7 & 184 & 20 & 143 & 10 & 46 & 10 \\
\hline 836.7 & 184 & 20 & 143 & 10 & 47 & 10 \\
\hline 837.7 & 184 & 20 & 143 & 10 & 47 & 10 \\
\hline 838.7 & 184 & 20 & 144 & 10 & 47 & 10 \\
\hline 839.7 & 184 & 20 & 144 & 10 & 47 & 10 \\
\hline 840.7 & 184 & 20 & 144 & 10 & 47 & 10 \\
\hline 841.7 & 184 & 20 & 144 & 10 & 47 & 10 \\
\hline 842.7 & 184 & 20 & 144 & 10 & 47 & 10 \\
\hline 843.7 & 184 & 20 & 145 & 10 & 47 & 10 \\
\hline 844.7 & 184 & 20 & 145 & 10 & 47 & 10 \\
\hline 845.7 & 184 & 20 & 145 & 10 & 47 & 10 \\
\hline 846.7 & 184 & 20 & 146 & 10 & 48 & 10 \\
\hline 847.7 & 184 & 20 & 146 & 10 & 48 & 10 \\
\hline 848.7 & 184 & 20 & 146 & 10 & 48 & 10 \\
\hline 849.7 & 184 & 20 & 146 & 10 & 48 & 10 \\
\hline 850.7 & 184 & 20 & 147 & 10 & 48 & 10 \\
\hline 851.7 & 184 & 20 & 147 & 10 & 48 & 10 \\
\hline 852.7 & 184 & 20 & 148 & 10 & 48 & 10 \\
\hline 853.7 & 184 & 20 & 148 & 10 & 48 & 10 \\
\hline
\end{tabular}




$\begin{array}{lllllll}854.7 & 184 & 20 & 148 & 10 & 48 & 10 \\ 855.7 & 184 & 20 & 148 & 10 & 48 & 10 \\ 856.7 & 184 & 20 & 149 & 10 & 48 & 10 \\ 857.7 & 184 & 20 & 149 & 10 & 48 & 10 \\ 858.7 & 184 & 20 & 141 & 10 & 46 & 10 \\ 859.7 & 184 & 20 & 141 & 10 & 46 & 10 \\ 860.7 & 184 & 20 & 150 & 10 & 49 & 10 \\ 861.7 & 184 & 20 & 150 & 10 & 49 & 10 \\ 862.7 & 184 & 20 & 150 & 10 & 49 & 10 \\ 863.7 & 184 & 20 & 150 & 10 & 49 & 10 \\ 864.7 & 184 & 20 & 151 & 10 & 49 & 10 \\ 865.7 & 184 & 20 & 151 & 10 & 49 & 10 \\ 866.7 & 184 & 20 & 152 & 10 & 50 & 10 \\ 867.7 & 184 & 20 & 152 & 10 & 50 & 10 \\ 868.7 & 184 & 20 & 152 & 10 & 50 & 10 \\ 869.7 & 184 & 20 & 152 & 10 & 50 & 10 \\ 870.7 & 184 & 20 & 153 & 10 & 50 & 10 \\ 871.7 & 184 & 20 & 153 & 10 & 50 & 10 \\ 872.7 & 184 & 20 & 153 & 10 & 50 & 10 \\ 873.7 & 184 & 20 & 153 & 10 & 50 & 10 \\ 874.7 & 184 & 20 & 154 & 10 & 50 & 10 \\ 875.7 & 184 & 20 & 154 & 10 & 50 & 10 \\ 876.7 & 184 & 20 & 154 & 10 & 50 & 10 \\ 877.7 & 184 & 20 & 154 & 10 & 50 & 10 \\ 878.7 & 184 & 20 & 154 & 10 & 50 & 10 \\ 879.7 & 184 & 20 & 154 & 10 & 50 & 10 \\ 880.7 & 184 & 20 & 154 & 10 & 50 & 10\end{array}$

